\title{
Fatores associados à demência em idosos
}

\author{
Factors associated with dementia in elderly
}

Camila de Souza dos Santos (http://orcid.org/0000-0002-1660-8657) ${ }^{1}$

Thaíssa Araujo de Bessa (http://orcid.org/0000-0002-7326-2832) ${ }^{1}$

André Junqueira Xavier (https://orcid.org/0000-0002-8282-0939) ${ }^{1}$

${ }^{1}$ Universidade Federal de Santa Catarina. R. Eng. Agrônomo Andrei Cristian Ferreira s/n, Trindade. 88040-900 Florianópolis SC Brasil.camila_sfisio@ hotmail.com

\begin{abstract}
We analyzed the factors associated with dementia in the elderly attended at a memory outpatient clinic of the University of Southern Santa Catarina (UNISUL). This is a cross-sectional study with data analysis of medical records from January 2013 to April 2016. The outcome was the clinical diagnosis of dementia. The control variables were: serum vitamin D level at the time of diagnosis, gender, skin color, schooling, age, type 2 diabetes, hypertension, and depression. We performed a crude and adjusted analysis with logistic regression. The sample consisted of 287 elderly, with the predominance of age between 60 and 69 years (48.78\%), female $(79.09 \%)$ and white $(92.33 \%)$. The mean number of years of study was 6.95 years (SD \pm 4.95) and mean vitamin $D$ was $26.09 \mathrm{ng} / \mathrm{mL}$ (SD $\pm 9,20)$. The prevalence of elderly with dementia was $16.72 \%$. Depression was the most prevalent (42.50\%) among the morbidities, followed by hypertension (31.71\%). The following were independently associated with dementia: vitamin $D$ $(\mathrm{OR}=0.92,95 \% \mathrm{CI}, 0.88 ; 0.97)$, depression $(\mathrm{OR}=$ 4.09, 95\%CI, 1.87;8.94), hypertension $(O R=2.65$, 95\%CI, 1.15;6.08) and individuals aged 80 years and over $(O R=3.9795 \% C I, 1.59 ; 9.91)$. Dementia prevalence was high and diagnosed dementia was associated with lower levels of vitamin D. Vitamin $D$ is a modifiable factor, opening up essential perspectives for public health policies.
\end{abstract}

Key words Elderly, Dementia, Memory outpatient clinic
Resumo Analisar os fatores associados à demência em idosos atendidos em um ambulatório de memória da Universidade do Sul de Santa Catarina (Unisul). Estudo transversal com análise de dados de prontuário no período de 01/2013 a 04/2016. O desfecho foi o diagnóstico clínico de demência. As variáveis de controle foram: nível sérico de vitamina $D$ na época do diagnóstico, sexo, cor da pele, escolaridade, idade, diabetes tipo 2, hipertensão arterial e depressão. Foi realizada análise bruta e ajustada com regressão logística. Amostra de 287 idosos, com predominância de idade entre 60 e 69 anos (48,78\%), sexo feminino (79,09\%), cor da pele branca (92,33\%). A média de anos de estudo foi de 6,95 anos (DP $\pm 4,95) e$ da vitamina $D 26,09 \mathrm{ng} / \mathrm{mL}(\mathrm{DP} \pm 9,20)$. A prevalência de idosos com demência foi de 16,72\%. Dentre as morbidades a depressão foi a de maior prevalência seguida pela hipertensão arterial. Estiveram independentemente associadas à demência: vitamina D (OR = 0,92 IC 95\% 0,88;0,97), depressão $(O R=4,09$ IC95\% 1,87;8,94), hipertensão arterial (OR = 2,65 IC95\% 1,15;6,08) e indivíduos com idade igual e maior que 80 anos. A prevalência de demência foi alta, e houve associação de níveis mais baixos de vitamina $D$ com diagnóstico de demência. Sendo a vitamina D um fator modificável, abrindo importantes perspectivas para políticas de saúde pública.

Palavras-chave Idoso, Demência, Ambulatório da memória 


\section{Introdução}

No Brasil, o envelhecimento populacional ocorre de modo acelerado. Prevê-se que a população com 60 anos ou mais, correspondente a $10 \%$ do total em 2010, atingirá $13,7 \%$ em 2020, chegando a $23,8 \%$ de idosos em 2040, ou seja, quase um quarto do total de habitantes do país ${ }^{1}$. Estas transformações na pirâmide etária são consequências das mudanças no balanço entre natalidade e mortalidade, bem como no perfil de morbidade da população, caracterizando a transição demográfica e epidemiológica.

Com aumento da expectativa de vida as doenças crônicas não transmissíveis (DCNT) destacam-se como importante desafio de saúde pública, principalmente pela alta morbidade que causam. Estas doenças podem provocar sérios graus de incapacidade que afetam tanto os hábitos de vida e o bem-estar do indivíduo, quanto à economia do país. Em 2002, as DCNT eram responsáveis por quase $60 \%$ de todas as mortes ${ }^{2}$. Aproximadamente $80 \%$ dos idosos possuem pelo menos uma doença crônica e, destes, 50\% apresentam duas ou mais patologias ${ }^{3}$.

Dentre as DCNT relacionadas ao envelhecimento estão as demências, que se destacam como causas principais de comprometimento funcional e da qualidade de vida do idoso 4 . Segundo a Organização Mundial de Saúde (OMS), em 2012 mais de mais de 35 milhões de pessoas no mundo apresentavam algum grau de demência, sendo que este número poderá triplicar até 2050, atingindo mais de 115 milhões de pessoas, a maior prevalência de demência recairá sobre países de baixa e média renda, com cerca de $60 \%$ dos casos de demência ${ }^{5}$.

De acordo com a $\mathrm{OMS}^{6}$, o tempo vivido com demência responde por $11,9 \%$ dos anos de convívio com incapacidades decorrentes de doenças não transmissíveis, apresentando, no ano de 2010, um custo mundial estimado em US\$ 604 bilhões.

As perdas cognitivas decorrentes das demências são mais prevalentes na população femini$\mathrm{na}^{7}$, entre indivíduos com baixa escolaridade ${ }^{8}$ que não praticam atividade física ${ }^{9}$, aqueles de baixa condição econômica ${ }^{10}$ com idade avançada ${ }^{11}$ e em situação de fragilidade ${ }^{12}$. Outros fatores de risco associados à demência são: hipertensão arterial $^{13}$ diabetes mellitus ${ }^{14}$ depressão ${ }^{15}$ e a baixos níveis de vitamina $\mathrm{D}^{16}$, sendo o último um fator modificável.

Nos últimos anos, as associações entre vitamina $\mathrm{D}$ e demência têm atraído interesse cres- cente ${ }^{16,17}$. Estudos indicam que a deficiência de vitamina $\mathrm{D}$ é mais prevalente em pacientes com demência ${ }^{18}$. Uma meta-análise mostrou que pessoas dementadas possuem menor nível de vitamina D (25-hidroxivitamina D (25 (OH) D)) comparadas com grupo controle de pessoas saudáveis pareadas por idade ${ }^{19}$. A baixa de $25(\mathrm{OH})$ $\mathrm{D}$ pode ser apenas um marcador ou potencial fator de risco de se desenvolver demência como apontam estudos recentes ${ }^{18-21}$.

No estudo de Baumgart et al..$^{22}$ destaca a importância de estudos que visem analisar fatores de risco modificáveis à demência, já que esses são passíveis de intervenção, destacando a prática de comportamentos saudáveis.

Desta forma o presente estudo tem como objetivo analisar os fatores associados à demência em idosos acompanhados de um Ambulatório de Memória da Universidade do Sul de Santa Catarina.

\section{Métodos}

Trata-se de um estudo transversal, realizado na cidade de Palhoça, Santa Catarina. Segundo o $\mathrm{IBGE}^{23}$, a cidade de Palhoça, é composta por aproximadamente 137.334 habitantes, destes, os indivíduos com idade igual e superior a 60 anos correspondem a 30.513 habitantes $(8,79 \%)$. Foram analisados dados a partir dos prontuários de idosos acompanhados pelo Ambulatório da Memória da Universidade do Sul de Santa Catarina (Unisul).

Foram identificados 406 prontuários, de janeiro de 2013 a abril de 2016. Destes, foram excluídos 119, pois os prontuários não apresentavam a dosagem sérica de vitamina $D$. A amostra final do estudo foi de 287 idosos, os critérios de inclusão foram indivíduos com idade igual e superior a 60 anos, com diagnóstico clínico do estado cognitivo e que haviam feito a dosagem sérica de 25-Hidroxivitamina D 25(OH)D na época do diagnóstico, como parte dos exames de rotina padronizados para a investigação de demências e suas causas. Os critérios de exclusão foram indivíduos que fizeram recentemente ou estavam fazendo reposição de vitamina $D$ no período estudado, portadores de delírium ou outras doenças agudas no momento da coleta dos exames ou do processo diagnóstico para demência.

Em relação à variável dependente, a demência foi analisada a partir do diagnóstico clínico, que foi composto de anamnese, exame físico, exame neurológico, exames complementares e avalia- 
ção cognitiva ${ }^{24,25}$. Os testes de avaliação cognitiva para rastreio e apoio ao diagnóstico utilizados foram o MoCA (Montreal Cognitive Assessment) e CDR (Clinical Dementia Rating), ambos validados no Brasil.

As variáveis independentes foram o nível sérico de vitamina $\mathrm{D}(25(\mathrm{OH}) \mathrm{D}) \mathrm{em} \mathrm{ng} / \mathrm{mL}$, idade (60 a 69, 70 a 79 e 80 anos e mais), sexo (masculino e feminino), cor da pele (brancos, pretos e pardos), escolaridade (anos completos de estudo) e em relação às variáveis de condição de saúde (diabetes mellitus, hipertensão arterial e depressão). Os dados foram obtidos a partir do diagnóstico clínico e foram dicotomizadas em não e sim.

A análise estatística foi realizada por meio do programa Stata/SE 13.0. Realizou-se a estatística descritiva, com frequência absoluta e relativa por meio do cálculo de medidas de tendência central e de dispersão para as variáveis contínuas, e de frequência para as variáveis categóricas. Para a identificação dos fatores associados à demência, inicialmente, foram realizadas análises bivariadas entre cada variável de exposição e a variável dependente. Foi aplicado o teste do qui-quadrado ( 2) de Pearson, com nível de significância de 5\%. Foram também obtidas as odds ratio ( $\mathrm{OR}$ não ajustada) entre variáveis dicotômicas e seus respectivos intervalos de confiança em nível de 95\% (IC95\%).

Foi realizada regressão logística multivariada, utilizando todas as variáveis da análise bivariada a fim de identificar as variáveis que se mantiveram significativamente relacionadas à ocorrência de demência $(p<0,05)$ e com plausibilidade biológica. Utilizou-se o teste de razão da máxima verossimilhança para verificar a significância do modelo final.

Este estudo foi aprovado pelo Comitê de Ética em Pesquisa com Seres Humanos (CEPSH) da Universidade Federal de Santa Catarina (UFSC), com co-participação da Universidade do Sul de Santa Catarina. Todos os participantes assinaram o termo de consentimento livre e esclarecido e em caso de vulnerabilidade, o responsável legal.

\section{Resultados}

A amostra foi composta por 287 idosos, com a idade entre 60 e 69 anos $(48,78 \%)$, do sexo feminino $(79,09 \%)$, de cor da pele branca $(92,33 \%)$. A prevalência de idosos com demência foi de $16,72 \%$, entre os idosos dementados a média dos níveis de vitamina $\mathrm{D}$ foi de $21,90(\mathrm{DP} \pm 8,10)$ e dos idosos não dementados de 26,93 ng/mL (DP $\pm 8,80)$.

Dentre as morbidades, a depressão foi a de maior prevalência $(42,50 \%)$ seguida pela hipertensão arterial $(31,71 \%)$ e diabetes mellitus (20,21\%). A média de anos de estudo foi de 6,95 anos $(\mathrm{DP} \pm 4,95)$ e da vitamina D 26,09 $\mathrm{ng} / \mathrm{mL}$ $(\mathrm{DP} \pm 9,20)$ (Tabela 1).

$\mathrm{Na}$ análise bivariada o aumento da vitamina $\mathrm{D}$ obteve efeito protetor em relação à demência $(\mathrm{OR}=0,93$ IC95\% 0,89;0,97). Em relação às morbidades, os idosos diagnosticados com depressão tiveram $166 \%$ de chance de ter o desfecho (OR = 2,66 IC95\% 1,40;5,05). Os indivíduos com 80 anos e mais tiveram $354 \%$ de chance de ter demência em relação àqueles com idade entre 60 e 69 anos (OR = 4,54 IC95\% 2,05;10,04) (Tabela 2).

$\mathrm{Na}$ análise ajustada, permaneceram independentemente associadas à demência a vitamina $\mathrm{D}$, a cada aumento de uma unidade de vitamina $\mathrm{D}(\mathrm{ng} / \mathrm{mL})$ diminui em $8 \%$ a chance de ter o desfecho (OR = 0,92 IC 95\% 0,88;0,97), os idosos diagnosticados com depressão $(\mathrm{OR}=$ 4,09 IC95\% 1,87;8,94) e hipertensão (OR = 2,68 IC95\% 1,15;6,08) tiveram mais chance de terem demência, 309\% e 168\%, respectivamente, quando comparados com os idosos que não apresentavam a doença. Em relação à idade, os indivíduos mais velhos com 80 anos e mais tiveram 297\% mais chance de serem diagnosticados com demência, quando comparados com aqueles de 60 a 69 anos (OR = 3,97 IC95\% 1,59;9,91) (Tabela 2). A partir do teste de razão da máxima verossimilhança, o modelo final foi considerado ajustado $(\mathrm{p}=0,39)$.

\section{Discussão}

Até o momento desta revisão, este é o primeiro estudo brasileiro que avalia os fatores associados à demência em idosos no âmbito de um ambulatório da memória. A prevalência de demência encontrada neste estudo foi de $16,72 \%$, superior à de Nitrini et al. que avaliaram estudos sobre prevalência de demência nos países da América Latina e encontrou uma prevalência de 7,1\%. Esta diferença ocorreu pela especificidade de uma população atendida em um ambulatório da memória que recebe pessoas encaminhadas da atenção básica já com suspeitas de problemas cognitivos $^{26}$.

No Brasil, poucos estudos avaliam a demência na população idosa, e os existentes apresen- 


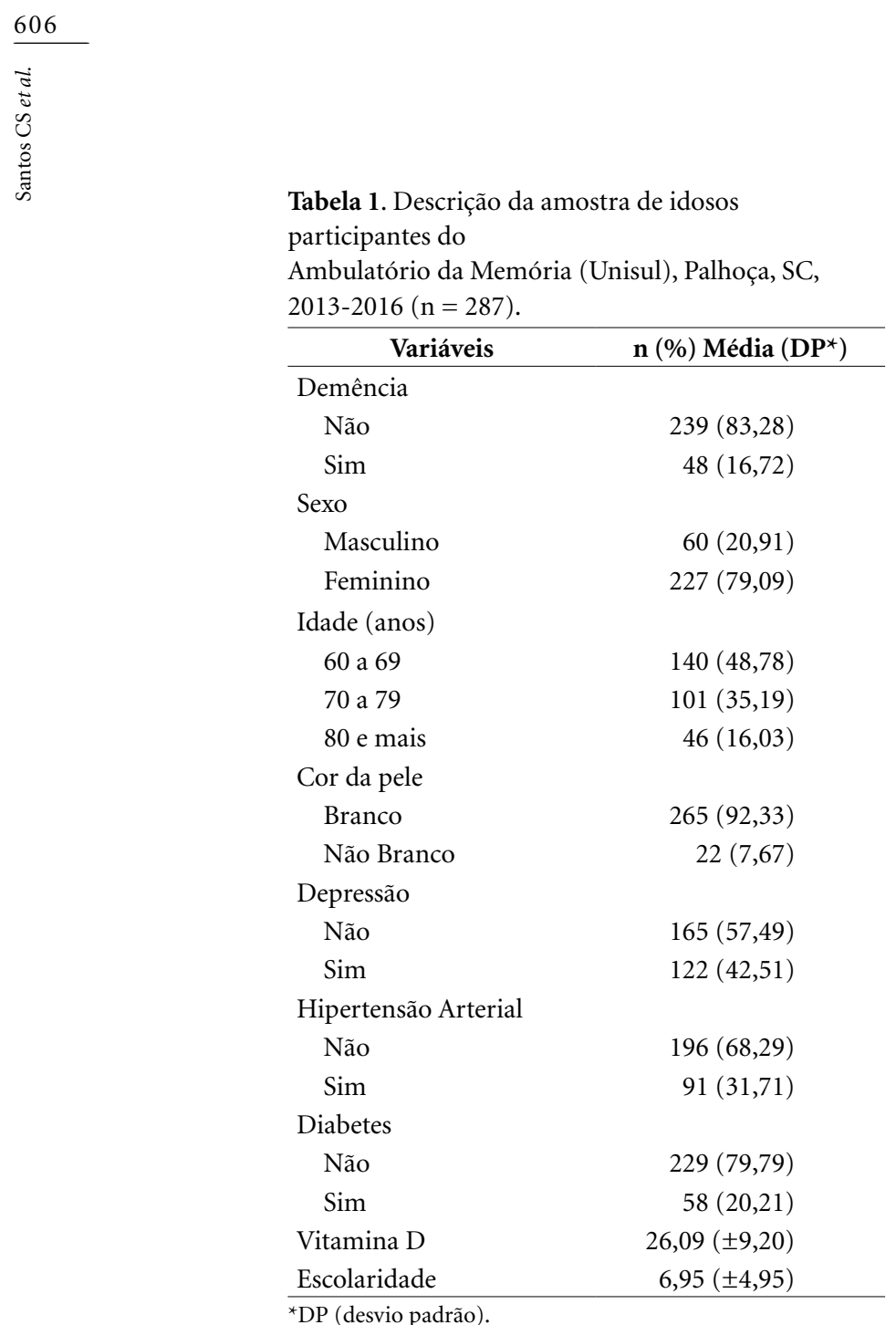

tam diferenças significativas em relação à prevalência e incidência. Em uma revisão sistemática ${ }^{27}$ recente a prevalência de demência entre idosos brasileiros variou entre $5,1 \%$ a $19 \%$, porém a maiorias dos estudos avaliaram o estado cognitivo por questionários como o Mini Exame do Estado Mental (MEEM), e não a partir do diagnóstico clínico (padrão ouro) como neste estudo.

Verificamos que a cada aumento de uma unidade de vitamina $\mathrm{D}(\mathrm{ng} / \mathrm{mL})$ diminui em $8 \%$ a prevalência de ter o diagnóstico de demência, recentes estudos têm demonstrado essas associações ${ }^{16,17,20,21}$. Em um estudo de coorte realizado com idosos franceses com acompanhamento de 12 anos verificou que idosos com insuficiência (20 a $29 \mathrm{ng} / \mathrm{mL})$ e deficiência $(<20 \mathrm{ng} / \mathrm{mL})$ de vitamina $\mathrm{D}$ tiveram aproximadamente 3 vezes maior risco de terem demência ${ }^{28}$.

Os resultados deste estudo corroboram com outros estudos experimentais que sugeriram que a hipovitaminose $\mathrm{D}$ poderia mediar proces- sos neurodegenerativos envolvidos nas demências $^{16,29,30}$. Estudos de caso controle indicaram que indivíduos com demência tinham menores concentrações circulantes de vitamina $\mathrm{D}^{31,32}$. Além disso, vários estudos longitudinais encontraram uma associação entre baixos níveis de vitamina $\mathrm{D}$ e declínio cognitivo acelerado ${ }^{30,33-36}$.

No entanto, existem resultados conflitantes, como o encontrado em dois estudos longitudinais realizados na Suécia e nos Estados Unidos (EUA). Nestes estudos não foram encontradas associações entre os níveis de vitamina D e o estado cognitivo de idosos. Porém, no estudo realizado na Suécia ${ }^{37}$, a amostra foi composta apenas de homens e a prevalência de hipovitaminose $\mathrm{D}$ foi baixa. Destes, apresentavam insuficiência (15,5\%) e deficiência (1,7\%) . Já no estudo realizado nos EUA $^{38}$, apesar de não encontrarem associações entre vitamina $\mathrm{D}$ e demência, verificaram que baixas concentrações de vitamina $\mathrm{D}$ foram associadas a pior função executiva, velocidade de processamento e habilidades visuo-perceptivas.

Sabe-se que a vitamina $\mathrm{D}$ tem um papel importante no desenvolvimento do cérebro e maturação dos receptores de vitamina D (VDRs) que estão presentes em várias áreas do cérebro, incluindo as relacionadas à aprendizagem e funções de memória, além disso, a vitamina $\mathrm{D}$ está envolvida em vários caminhos para a saúde do cérebro, incluindo neurotransmissão, neuroproteção, modulação de resposta imune, inibição dos agentes pró-inflamatórios e regulação de estresse oxidativo ${ }^{16,30,39}$.

Foram realizados para verificar o efeito da suplementação de vitamina $\mathrm{D}$ na função cognitiva, porém Annweiler et al. ${ }^{40}$ observaram que, após a adição de vitamina D de $800 \mathrm{IU} /$ dia em idosos com declínio cognitivo, houve melhora na função cognitiva. O consumo de 800 UI de vitamina $\mathrm{D}$ por dia resultou em redução no risco de $\mathrm{Al}$ zheimer após 7 anos de acompanhamento ${ }^{41}$. Este efeito neuroprotetor foi confirmado ainda por estudo experimental que relatou melhora cognitiva $^{42}$. Os benefícios da suplementação aparecem a partir de 4 semanas ${ }^{43}$ e parecem ser particularmente fortes para função executiva e velocidade de processamento ${ }^{44}$.

Em relação às morbidades, aproximadamente $50 \%$ dos idosos da amostra apresentavam o diagnóstico de depressão, Barcelos-Ferreira et al. ${ }^{45}$ revisaram a literatura científica sobre depressão em idosos da comunidade no Brasil e identificaram uma prevalência de depressão de $7 \%$ e de sintomas depressivos, de $26 \%$. Estudo feito com idosos da comunidade no Canadá encontrou 
Tabela 2. Análise bruta e ajustada das variáveis associadas à demência, de idosos participantes de um Ambulatório da Memória, Palhoça, SC, 2013-2016.

\begin{tabular}{|c|c|c|c|c|c|}
\hline \multirow{2}{*}{ Variáveis } & \multirow[b]{2}{*}{$\%$} & \multicolumn{2}{|c|}{ Análise Bruta } & \multicolumn{2}{|c|}{ Análise Ajustada } \\
\hline & & OR $(\mathrm{IC} 95 \%)^{*}$ & p-valor & OR $($ IC95\%)* & p-valor \\
\hline Vitamina D & & $0,93(0,89 ; 0,97)$ & 0,001 & $0,92(0,88 ; 0,97)$ & 0,001 \\
\hline \multicolumn{6}{|l|}{ Sexo } \\
\hline Masculino & 20,00 & 1,00 & & 1,00 & \\
\hline Feminino & 15,85 & $0,75(0,36 ; 1,55)$ & 0,446 & $0,42(0,17 ; 1,02)$ & 0,060 \\
\hline \multicolumn{6}{|l|}{ Idade (anos) } \\
\hline 60 a 69 & 11,43 & 1,00 & & 1,00 & \\
\hline 70 a 79 & 14,85 & $1,35(0,63 ; 2,88)$ & 0,435 & $1,61(0,72 ; 3,62)$ & 0,247 \\
\hline 80 e mais & 36,95 & $4,54(2,05 ; 10,04)$ & $<0,001$ & $3,97(1,59 ; 9,91)$ & 0,003 \\
\hline \multicolumn{6}{|l|}{ Cor da pele } \\
\hline Branco & 17,35 & 1,00 & & 1,00 & \\
\hline Pretos/Pardos & 9,10 & $0,47(0,11 ; 2,10)$ & 0,318 & $0,54(0,11 ; 2,58)$ & 0,439 \\
\hline Escolaridade & & $0,95(0,88 ; 1,02)$ & 0,138 & $0,95(0,88 ; 1,03)$ & 0,260 \\
\hline \multicolumn{6}{|l|}{ Depressão } \\
\hline Não & 10,90 & 1,00 & & 1,00 & \\
\hline Sim & 24,60 & $2,66(1,40 ; 5,05)$ & 0,003 & $4,09(1,87 ; 8,94)$ & $<0,001$ \\
\hline \multicolumn{6}{|c|}{ Hipertensão Arterial } \\
\hline Não & 15,81 & 1,00 & & 1,00 & \\
\hline Sim & 18,68 & $1,22(0,64 ; 2,34)$ & 0,545 & $2,68(1,16 ; 6,18)$ & 0,020 \\
\hline \multicolumn{6}{|l|}{ Diabetes } \\
\hline Não & 14,85 & 1,00 & & 1,00 & \\
\hline Sim & 24,14 & $1,82(0,90 ; 3,68)$ & 0,090 & $2,12(0,91 ; 4,95)$ & 0,080 \\
\hline
\end{tabular}

prevalência de depressão entre $1,3 \%$ e $18,8 \%$ nas mulheres e entre 0,9 e $7,9 \%$ em homens ${ }^{46}$.

Os idosos com depressão tiveram 4,09 vezes mais chance de terem demência, em recente estudo longitudinal de 14 anos mostrou que homens idosos com depressão tiveram maior chance de terem demência $(\mathrm{OR}=1,5 \text { IC95\% } 1,2 ; 2,0)^{47}$. Norton et al. estimaram que em 5 a $11 \%$ dos casos de doença de Alzheimer podem ser atribuídos à depressão, isso significa que a prevalência de demência na população seria reduzida pela mesma quantidade se a depressão puder ser prevenida ou adequadamente tratada ${ }^{48}$.

Por outro lado, em estudo realizado por Mirza et al. em 2014, com 4393 idosos, foram seguidos por até 13,7 anos para demência incidente e constatou que cerca de 13\% desenvolveram demência e que a depressão aumentou o risco de demência em aproximadamente $20 \%$ por 2 e 5 anos, mas o mesmo estudo sugere que os sintomas depressivos em idades avançadas são parte dos sintomas iniciais de demência e não um fator de risco independente para a mesma ${ }^{49}$. Há evidência de que a depressão pode levar a perda de volume do hipocampo, particularmente quando os sintomas são persistentes, contribuindo assim para o aparecimento da demência ${ }^{50}$.

Em relação à hipertensão arterial, após a análise ajustada, os idosos com hipertensão apresentaram 168\% maior chance de terem demência. Corroborando com estudo realizado em pessoas acima de 80 anos, na China verificou-se que os idosos com hipertensão tiveram 193\% mais chance de apresentarem transtorno cognitivo leve, e evoluir à demência ${ }^{51}$.

Curiosamente, em pessoas de 60 anos ou mais, a pressão arterial sistólica (PAS) foi inversamente associada à demência por todas as causas $^{52}$, esta associação inversa é paradoxal porque é geralmente reconhecida como um fator de risco para declínio cognitivo e demência ${ }^{53-55}$. No entanto, a associação entre pressão arterial e cérebro é complexa e depende de fatores como idade, cronicidade e uso de medicação anti-hipertensiva ${ }^{52}$.

As terapias anti-hipertensivas podem reduzir o declínio cognitivo e a incidência de demência, a maioria dos estudos observacionais sugeriram um potencial efeito preventivo das terapias anti 
-hipertensivas sobre declínio cognitivo e demência, particularmente bloqueadores dos canais de cálcio e bloqueadores do sistema renina-angiotensina ${ }^{56}$.

Não foi encontrada neste estudo associação entre diabetes mellitus e demência, possivelmente em função do tamanho da amostra, porém em uma meta-análise, o risco relativo de demência para pacientes com diabetes foi estimado em 1,46, com base em 6 estudos que reúnem um total de 5.706 pessoas com diabetes e 36.191 sem diabetes, analisando o risco relativo para a demência vascular foi de 2,48 , ou seja houve um considerável aumento do risco para este tipo de demência ${ }^{57}$.

A prevalência de mulheres no estudo foi de $79,09 \%$ isto esta associado com a feminização na velhice, as mulheres representam $55,5 \%$ da população idosa brasileira e $61 \%$ do contingente de idosos acima de 80 anos $^{23}$. Essa sobrerrepresentação feminina resulta da maior expectativa de vida das mulheres que, em média, vivem 8 anos a mais que os homens ${ }^{58}$.

A faixa etária com 80 anos e mais teve $297 \%$ maior chance para demência, quando comparada com a faixa de 60 a 69 anos, vários estudos ${ }^{26,27,59}$ tem demonstrado essa relação em que indivíduos mais velhos tem maior probabilidade de terem demência. Em relação à variável sexo não houve associação com o desfecho, porém muitos estu$\operatorname{dos}^{26,27}$ apontam que pessoas do sexo feminino apresentam maior chance de terem o desfecho.

As demências tem grande impacto nos custos da sociedade e da família, Veras et al. ${ }^{60}$ ao investigarem 41 famílias de portadores de demência residentes no município do Rio de Janeiro, constataram que a projeção de custos associados aos cuidados de idosos com demência atingia cerca de dois terços da renda familiar, encontrando-se um amento de $75 \%$ quando os idosos estavam nos estágios iniciais da doença e $80 \%$ quando outras doenças crônicas foram levadas em consideração.

Em 1998, Meek et al. ${ }^{61}$ afirmaram que em termos de custos totais para a sociedade, a DA era a terceira doença mais cara nos EUA, logo após o câncer e as doenças coronarianas. E ainda,
Brookmeyer et al. ${ }^{62}$ citaram que o impacto dos custos advindos do tratamento relacionados aos cuidados de saúde devido à DA seria enorme, trazendo uma marca para a economia dos EUA de até $\mathrm{U} \$ 36$ bilhões por ano.

Estimativas recentes revelaram que os custos associados à demência no Reino Unido são de 17 bilhões de euros por ano, custo que está estimado em 50 bilhões de euros para 2038, com incidência de 1,4 milhões de novos casos por ano ${ }^{63}$. O custo social dos cuidados da DA aumenta drasticamente com o aumento da severidade da doença, sendo a institucionalização o principal motivo ${ }^{64}$.

Citamos como as limitações deste estudo que não houve controle em relação a qual estação do ano os exames para dosagem da vitamina D foram realizados assumindo-se que houve uma distribuição aleatória ao longo do período estudado. Por se tratar de um estudo transversal não se pode inferir causalidade. A população estudada não permite generalização dos resultados para a população como um todo, pois se trata de um ambulatório especializado.

\section{Conclusão}

As demências possuem origem multifatorial e são consideradas um problema de saúde pública com grande impacto nos gastos de saúde. Neste estudo os fatores associados à demência foram vitamina $\mathrm{D}$, depressão, hipertensão arterial e idade acima dos 80 anos. Conhecer e entender esses fatores auxiliam na clínica médica, no diagnóstico e no tratamento de idosos dementados.

Os resultados deste trabalho podem influenciar positivamente políticas de saúde pública onde mudanças de hábito de vida praticamente sem custo como maior exposição solar podem resultar em melhores condições de saúde para os idosos, tanto pela possível proteção contra demência quanto pela prevenção da hipovitaminose $\mathrm{D}$ entre os dementados evitando maiores problemas de saúde. Para continuidade deste trabalho serão necessários estudos prospectivos, randomizados e de intervenção com amostras maiores. 


\section{Colaboradores}

CS Santos: concepção do estudo, análise estatística, redação do manuscrito. AJ Xavier: redação de trechos do manuscrito, planejamento das análises e revisão de sua versão final. TA Bessa: aporte teórico, revisão da versão final do manuscrito.

\section{Referências}

1. Mendes ACD, Sá DA, Miranda GMD, Lyra TM, Tavares RAW. Assistência pública de saúde no contexto da transição demográfica brasileira: exigências atuais e futuras. Cad Saude Publica 2012; 28(5):955-964.

2. Monteiro CA, Moura EC, Jaime PC, Lucca A, Florindo AA, Figueiredo ICR, Bernal R, Silva NN. Monitoramento de fatores de risco para doenças crônicas por meio de entrevistas telefônicas: métodos e resultados no município de São Paulo. Rev Saude Publica 2005; 39(1):47-57.

3. Hakansson K, Rovio S, Helkala EL, Vilska AR, Winblad B, Soininen H, Nissinen A, Mohammed AH, Kivipelto $\mathrm{M}$. Association between mid-life marital status and cognitive function in later life: population based cohort study. BMJ 2009; 339:b2462

4. Ballard C, Gauthier S, Corbett A, Brayne C, Aarsland D, Jones E. Alzheimer's disease. Lancet 2011; 377(9770):1019-1031.

5. World Health Organization (WHO). Dementia: a public health priority. Geneva: WHO; 2012.

6. World Health Organization (WHO). The global burden of disease: 2004 update. Geneva: WHO; 2008.

7. Santos AA, Mansano-Schlosser TCS, Ceolim MF, Pavarini SCI. Sono, fragilidade e cognição:estudo multicêntrico com idosos brasileiros. Rev Bras Enferm 2013; 66(3):351-357.

8. Trindade APNT, Barboza MA, Oliveira FB, Borges APO. Repercussão do declínio cognitiva na capacidade funcional em idosos institucionalizados e não institucionalizados. Fisioter Mov 2013; 26(2):281-289.

9. Freitas DHM, Campos FCA, Linhares LQ, Santos CR, Ferreira CB, Diniz BS, Tavares A. Autopercepção da saúde e desempenho cognitivo em idosos residentes na comunidade. Rev Psiquiatr Clín 2010; 37(1):32-35.

10. Macêdo AML, Cerchiari EAN, Alvarenga MRM, Faccenda O, Oliveira MAC. Avaliação funcional de idosos com déficit cognitivo. Acta Paul Enferm 2012; 25(3):358-363.

11. Castro-Costa E, Dewey ME, Uchôa E, Firmo JO, Lima-Costa MF, Stewart R. Trajectories of cognitive decline over 10 years in a Brazilian elderly population: the Bambuí cohort study of aging. Cad Saude Publica 2011; 27(Supl. 3):345-350.

12. Faria EC, Silva SA, Farias KRA, Cintra A. Avaliação cognitiva de pessoas idosas cadastradas na estratégia saúde da família: município do Sul de Minas. Rev Esc Enferm USP 2011; 45(2):1748-1752.

13. Banhato EFC, Guedes DV. Cognição e hipertensão: influência da escolaridade. Estud Psicol 2011; 28(2):143-151.

14. Almeida-Pititto B, Almada Filho CM, Cendoroglo MS. Déficit cognitivo: mais uma complicação do diabetes melito? Arq Bras Endocrinol Metab 2008; 52(7):1076-1083.

15. Trentini CM, Werlang BSG, Xavier FMF, Argimon IIL. A relação entre variáveis de saúde mental e cognição em idosos viúvos. Psicol Reflex Crít 2009; 22(2):236243.

16. Gezen-Ak D, Yllmazer S, Dursun E. Why vitamin D in Alzheimer's disease? The hypothesis. J Alzheimers Dis 2014; 40(2):257-269. 
17. Morley JE. Dementia: does vitamin D modulate cognition? Nat Rev Neurol 2014; 10(11):613-614.

18. Evatt ML, DeLong MR, Khazai N, Rosen A, Triche S, Tangpricha V. Prevalence of vitamin D insufficiency in patients with Parkinson disease and Alzheimer disease. Arch Neurol 2008; 65(10):1348-1352.

19. Zhao Y, Sun Y, Ji HF, Shen L. Vitamin D levels in Alzheimer's and Parkinson's diseases: a meta-analysis. Nutrition 2013; 29(6):828-832.

20. Littlejohns TJ, Henley WE, Lang IA, Annweiler C, Beauchet O, Chaves PH, Fried L, Kestenbaum BR, Kuller LH, Langa KM, Lopez OL, Kos K, Soni M, Llewellyn DJ. Vitamin D and the risk of dementia and Alzheimer disease. Neurology 2014; 83(10):920-928.

21. Afzal S, Bojesen SE, Nordestgaard BG. Reduced 25-hydroxyvitamin D and risk of Alzheimer's disease and vascular dementia. Alzheimers Dement 2014; 10(3):296-302.

22. Baumgart M, Snyder HM, Carrillo MC, Fazio S, Kim $\mathrm{H}$, Johns $\mathrm{H}$. Summary of the evidence on modifiable risk factors for cognitive decline and dementia: A population-based perspective. Alzheimer's \& Dementia $2015 ; 11(6): 718-726$.

23. Instituto Brasileiro de Geografia e Estatística (IBGE). Estimativas populacionais enviadas para o TCU, estratificadas por idade e sexo pelo MS/SGEP/Datasus. Rio de Janeiro: IBGE; 2012.

24. Parmera JB, Nitrini R. Investigation and diagnostic evaluation of a patient with dementia. Rev Med 2015; 94(3):179-184.

25. Nitrini R, Caramelli P, Bottino CMC, Damasceno BP, Brucki SMD, Anghinah R. Diagnóstico de Doença de Alzheimer no Brasil. Critérios diagnósticos e exames complementares. Arq Neuropsiquiatr 2005; 63(3):713719.

26. Nitrini R, Bottino CM, Albala C, Custodio Capuñay NS, Ketzoian C, Llibre Rodriguez JJ, Maestre GE, Ramos-Cerqueira AT, Caramelli P. Prevalence of dementia in Latin America: a collaborative study of population-based cohorts. Int Psychogeriatr 2009; 21(4) :622-630.

27. Fagundes SD, Silva MT, Theer MFRS, et al. Prevalence of dementia among elderly Brazilians: a systematic review. Sao Paulo Med J 2011; 129(1):46-50.

28. Feart C, Helmer C, Merle B, Pereira MG. Associations of lower vitamin $\mathrm{D}$ concentrations with cognitive decline and long-term risk of dementia and Alzheimer's disease in older adults. Alzheimer's \& Dementia 2017; 129(1):46-50.

29. Annweiler C, Bartha R, Goncalves S, Karras SN3, Millet P4, Féron F4, Beauchet O. Vitamin D-related changes in intracranial volume in older adults:A quantitative neuroimaging study. Maturitas 2015; 80(3):312-317.

30. Landel V, Annweiler C, Millet P, Morello M, Féron F. Vitamin D. Cognition and Alzheimer's disease: the therapeutic benefit is in the D-Tails. J Alzheimers Dis 2016; 53(2):419-444.
31. Annweiler C, Llewellyn DJ, Beauchet O. Low serum vitamin D concentrations in Alzheimer's disease: a systematic review and meta-analysis. J Alzheimers Dis 2013; 33(3):659-674.

32. Balion C, Griffith LE, Strifler L, Henderson M, Patterson C, Heckman G, Llewellyn DJ, Raina P. Vitamin D, cognition, and dementia: a systematic review and meta-analysis. Neurology 2012; 79(13):1397-1405.

33. Miller JW, Harvey DJ, Beckett LA, Green R, Farias ST, Reed BR, Olichney JM, Mungas DM, DeCarli C. Vitamin D status and rates of cognitive decline in a multiethnic cohort of older adults. JAMA Neurol 2015; 72(11):1295-1303.

34. Moon JH, Lim S, Han JW, Kim KM, Choi SH, Kim KW, Jang HC. Serum 25-hydroxyvitamin D level and the risk of mild cognitive impairment and dementia: the Korean Longitudinal Study on Health and Aging (KLoSHA). Clin Endocrinol (Oxf) 2015; 83(1):36-42.

35. Annweiler C, Montero-Odasso M, Llewellyn DJ, Richard-Devantoy S, Duque G, Beauchet O. Meta-analysis of memory and executive dysfunctions in relation to vitamin D. J Alzheimers Dis 2013; 37(1):147-171.

36. Overman MJ, Pendleton N, O’Neill TW, Bartfai G, Casanueva FF, Finn JD, Forti G, Rastrelli G, Giwercman A, Han TS, Huhtaniemi IT, Kula K, Lean MEJ, Punab M, Lee DM, Correa ES, Ahern T, Verschueren SMP, Antonio L, Gielen E, Rutter MK, Vanderschueren D, Wu FCW, Tournoy J; EMAS Study Group. Evaluation of cognitive subdomains, 25- hydroxyvitamin $\mathrm{D}$, and 1,25-dihydroxyvitamin $\mathrm{D}$ in the European Male Ageing Study. Eur J Nutr 2016; 56(6):2093-2103.

37. Olsson E, Byberg L, Karlstrom B, Cederholm T, Melhus $\mathrm{H}$, Sjögren P, Kilander L. Vitamin D is not associated with incident dementia or cognitive impairment: an 18-y follow-up study in community-living old men. Am J Clin Nutr 2017; 105(4):936-943.

38. Karakis I, Pase MP, Beiser A, Booth SL, Jacques PF, Rogers G, DeCarli C, Vasan RS, Wang TJ, Himali JJ3, Annweiler C, Seshadri S. Association of serum vitamin $\mathrm{D}$ with the risk of incident dementia and subclinical indices of brain aging: The Framingham Heart Study. J Alzheimers Dis 2016; 51(2):451-461.

39. Brouwer-Brolsma EM, de Groot LC. Vitamin D and cognition in older adults: an update of recent findings. Curr Opin Clin Nutr Metab Care 2015; 18:11-16.

40. Annweiler C, Fantino B, Gautier J, Beaudenon M, Thiery S, Beauchet O. Cognitive effects of vitamin D supplementationin older outpatients visiting a memory clinic: a pre-post study. J Am Geriatr Soc 2012; 60(4):793-795.

41. Annweiler C, Rolland Y, Schott AM, Blain H, Vellas B, Herrmann FR, Beauchet O. Higher vitamin D dietary intake is associated with lower risk of Alzheimer's disease: a 7-year follow-up. J Gerontol A Biol Sci Med Sci 2012; 67(11):1205-1211.

42. Stein MS, Scherer SC, Ladd KS, Harrison LC. A randomized controlled trial of high-dose vitamin D2 followed by intranasal insulin in Alzheimer's disease. $J$ Alzheimers Dis 2011; 26(3):477-484. 
43. Prybelski R, Agrawal S, Krueger D, Engelke JA, Walbrun F, Binkley N. Rapid correction of low vitamin D status in nursing home residents. Osteoporos Int 2008; 19(11):1621-1628.

44. Assmann KE, Touvier M, Andreeva VA, Deschasaux M, Constans T, Hercberg S, Galan P, Kesse-Guyot E. Midlife plasma vitamin D concentrations and performance in different cognitive domains assessed 13 years later. Br J Nutr 2015; 113(10):1628-1637.

45. Barcelos-Ferreira R, Izbicki R, Steffens DC, Bottino $\mathrm{CM}$. Depressive morbidity and gender in community-dwelling Brazilian elderly: systematic reviewand meta-analysis. Int Psychogeriatr 2010; 22(5):712-726.

46. Ostbye T, Kristjansson B, Hill G, Newman SC, Brouwer RN, McDowell I. Prevalence and predictors of depression in elderly Canadians: the canadian study of health and aging. Chronic Dis Can 2005; 26(4):93-99.

47. Almeida OP, Hankey GJ, Yeap BB, Golledge J, Flicker L. Depression as a modifiable factor to decrease the risk of Dementia. Transl Psychiatry 2017; 7(5):e1117.

48. Norton S, Matthews FE, Barnes DE, Yaffe K, Brayne C. Potential for primary prevention of Alzheimer's disease: an analysis of population-based data. Lancet Neurol 2014; 13(8):788-794.

49. Mirza SS, de Bruijn RF, Direk N, Hofman A, Koudstaal PJ, Ikram MA, Tiemeier H. Depressive symptoms predict incident dementia during short- but not long-term followup period. Alzheimers Dement 2014; 10:S323-S329.e321.

50. Taylor WD, McQuoid DR, Payne ME, Zannas AS, MacFall JR, Steffens DC. Hippocampus atrophy and the longitudinal course of late-life depression. Am J Geriatr Psychiatry 2014; 22(12):1504-1512.

51. Hai S, Dong B, Liu Y, Zou Y. Occurrence and risk factors of MCI in the older. Int J Geriatr Psychiatry 2012; 27(7):703-708.

52. Gabin JM, Tambs K, Saltvedt I, Sund E, Holmen J. Association between blood pressure and Alzheimer disease measured up to 27 years prior to diagnosis: the HUNT Study. Alzheimer's Research \& Therapy 2017; 9:37

53. Kivipelto M, Helkala EL, Laakso MP, Hänninen T, Hallikainen M, Alhainen K, Soininen H, Tuomilehto J, Nissinen A. Midlife vascular risk factors and Alzheimer's disease in later life: longitudinal, population based study. BMJ 2001; 322(7300):1447-1451.

54. Launer LJ, Ross GW, Petrovitch H, Masaki K, Foley D, White LR, Havlik RJ. Midlife blood pressure and dementia: the Honolulu-Asia aging study. Neurobiol Aging 2000; 21(1):49-55.

55. Skoog I, Lernfelt B, Landahl S, Palmertz B, Andreasson LA, Nilsson L, Persson G, Odén A, Svanborg A. 15-year longitudinal study of blood pressure and dementia. Lancet 1996; 347(9009):1141-1145.

56. Hernandorena I, Duron E, Vidal JS, Hanon O. Treatment options and considerations for hypertensive patients to prevent dementia. Expert Opin Pharmacother 2017; 29(10):1-12.
57. Cheng G, Huang C, Deng H, Wang H. Diabetes as a risk factor for dementia and mild cognitive impairment: A meta-analysis of longitudinal studies. Intern Med J 2012; 42(5):484-491.

58. Kuchemann BA. Envelhecimento populacional, cuidado e cidadania: velhos dilemas e novos desafios. Revista Sociedade e Estado 2012; 27(1):165-180.

59. Holz AW, Nunes BO, Thumé E, Lange C, Facchini LA. Prevalência de déficit cognitivo e fatores associados entre idosos de Bagé, Rio Grande do Sul, Brasil. Rev Bras Epidemiol 2013; 16(4):880-888.

60. Veras BP, Caldas CP, Dantas S, Sancho LG, Sicsú B, Motta LB. Demented elderly people living at home in Rio de Janeiro, Brazil: Evaluation of expenditure care. Psychogeriatrics 2008; 8(2):88-95.

61. Meek PD, McKeithan K, Schumock GT. Economic considerations in Alzheimer's disease. Pharmacotherapy 1998; 18(Pt. 2):68-73.

62. Brookmeyer R, Gray S, Kawas C. Projections of Alzheimer's disease in the United States and the public health impact of delaying disease onset. Am J Public Health 1998; 88(9):1337-1342.

63. McCallion H. Dementia Training. Mental Health Practice 2009; 12(7):8.

64. Hux MJ, O’Brien BJ, Iskedjian M, Goeree R, Gagnon M, Gauthier S. Relation between severity of Alzheimer's disease and costs of caring. CMAJ 1998; 159(5):457-465.

Artigo apresentado em 21/03/2018

Aprovado em 16/05/2018

Versão final apresentada em 18/05/2018 
